\title{
Using Finite Element and Contour Method to Evaluate Residual Stress in Thick Ti-6Al-4V Alloy Welded by Electron Beam Welding
}

\author{
Pu Xie $^{1,2} \cdot$ Hai-Yan $\mathrm{Zhao}^{1,2} \cdot{\text { Bing } \mathrm{Wu}^{3} \cdot \text { Shui-Li Gong }}^{3}$
}

Received: 31 August 2014/Revised: 1 April 2015/Published online: 1 May 2015

(c) The Chinese Society for Metals and Springer-Verlag Berlin Heidelberg 2015

\begin{abstract}
This work was to reveal the residual stress profile in electron beam welded Ti-6Al-4V alloy plates $(50 \mathrm{~mm}$ thick) by using finite element and contour measurement methods. A three-dimensional finite element model of 50-mmthick titanium component was proposed, in which a column-cone combined heat source model was used to simulate the temperature field and a thermo-elastic-plastic model to analyze residual stress in a weld joint based on ABAQUS software. Considering the uncertainty of welding simulation, the computation was calibrated by experimental data of contour measurement method. Both test and simulated results show that residual stresses on the surface and inside the weld zone are significantly different and present a narrow and large gradient feature in the weld joint. The peak tensile stress exceeds the yield strength of base materials inside weld, which are distinctly different from residual stress of the thin Ti-6Al-4V alloy plates presented in references before.
\end{abstract}

KEY WORDS: Electron beam welding; Residual stress; Finite element method; Contour method; Titanium alloy

\section{Introduction}

Titanium, such as Ti-6Al-4V alloy, has been widely used in the aircraft industry due to its combination of corrosion resistance, heat resistance and high specific strength [1]. Electron beam welding (EBW) is always used to weld titanium alloy structures because it can generate high concentrated heat input which can weld those thick bearing

Available online at http://link.springer.com/journal/40195

Hai-Yan Zhao

hyzhao@tsinghua.edu.cn

1 Department of Mechanical Engineering, Tsinghua University, Beijing 100084, China

2 State Key Laboratory of Tribology, Tsinghua University, Beijing 100084, China

3 Beijing Aeronautical Manufacture Technology Research Institute, Beijing 100024, China capacity titanium alloy structures in aircraft and has a narrow fusion zone. However, just like any other fusion weld technology, the regions near the weld line undergo several thermal cycles. The non-uniform heating and cooling in the material consequently induce inhomogeneous plastic deformation and residual stress in the welded joint. The welding-induced tensile residual stress may cause brittle fracture, buckling deformation and stress corrosion cracking, resulting in the reduction of fatigue life of welded structures in service [2].

The welded residual stress of 50-mm-thick Ti-6Al-4V EBW plates is complex due to its intense heat input energy and large structure dimensions. In recent years, the finite element method (FEM) has developed as an important tool for evaluating the welded residual stress. A number of finite element models have been proposed to investigate temperature fields, residual stress and deformation of welding process. Goldak et al. [3] used FEM to compute temperature field for several welding parameters in arc welding. Deng et al. [4] computed the welded stress in low- 
carbon-alloyed steel by taking the effect of the solid-state phase transformations into account. Lacki et al. [5] used FEM and experimental method to analyze the influence of parameters on geometry of fusion zone and distribution of residual stresses in EBW of high-strength steel, and the calculated residual stresses were compared with X-ray diffraction measurement results. Liu et al. [6] used a three-dimensional (3D) thermal elastic-plastic finite element model to predict welded residual stresses induced by full-penetration laser welding of Ti-6Al-4V alloy $(4 \mathrm{~mm}$ thick), and residual stress measurements by hole-drilling method were also performed to validate the simulated results.

In general, the physical and chemical reactions involved in EBW are always complicated, and there are still many uncertainties of the computed parameters in FEM such as the thermal and mechanical properties of materials at hightemperature zone [7]. The accurate prediction of residual stresses is difficult and always necessary to calibrate the simulated results by some experimental measurements. The X-ray diffraction method and hole-drilling method only can be used to measure the surface stress, which are not suitable for measuring internal stresses of thick plates in current work. Some typical techniques for instance neutron diffraction, deep hole-drilling and contour method could be used to measure the variation of residual stresses through the thickness [8]. The contour technique has been identified as the most powerful method for thick component, which is particular in providing a two-dimensional (2D) map of residual stress over a cross section. [9-11].

Even though a number of numerical models have been developed to predict welded residual stresses, there are limited models that have been developed to simulate thicksection electron beam welded Ti-6Al-4V alloy plates (50 $\mathrm{mm}$ thick). In the present work, the emphasis is laid on using both FEM and contour technologies to investigate the welded residual stress. Considering the uncertainty of welding simulation, the computational results are confirmed by experimental data from contour measurement method.

\section{Experimental}

\subsection{Test Component Preparation}

The material studied in present work is Ti-6Al-4V titanium alloy with composition (wt\%) of $\mathrm{Al} \mathrm{6.5,} \mathrm{V} \mathrm{4.25,} \mathrm{Fe} \mathrm{0.04,} \mathrm{C}$ $0.02, \mathrm{~N} 0.015, \mathrm{H} 0.0018$ and balance of Ti. Ti-6Al-4V is a near $\alpha$-Ti titanium alloy, whose melt point is $\sim 1660{ }^{\circ} \mathrm{C}$, yield strength is $890 \mathrm{MPa}$, Young's modulus is $112 \mathrm{GPa}$, and the Poisson's ratio is 0.34 [12]. The plates were cut by wire electric discharge machining (EDM) from the Ti-6Al-
4V block, which was obtained by forging. The plate surface was polished by milling before welding. All the plates were heated to $650{ }^{\circ} \mathrm{C}$ and held for $2 \mathrm{~h}$ to remove the initial residual stress, and then furnace was cooled down to room temperature.

A single-pass EBW was processed by CV65M EBW system in the $3.0 \times 10^{-2} \mathrm{~Pa}$ vacuum. A welding voltage of $150 \mathrm{kV}$, a welding current of $128 \mathrm{~mA}$, and a welding speed of $800 \mathrm{~mm} / \mathrm{min}$ were used in the EBW for the purpose of penetrating entirely, and the electron beam was focused below the top surface of the component.

The Ti-6Al-4V alloy plates with dimension of $200 \mathrm{~mm} \times 150 \mathrm{~mm} \times 50 \mathrm{~mm}$ were used as the components and subjected to fully penetrated EBW along $x$-direction in the mid-width, as shown in Fig. 1. The $x, y$ and $z$ direction separately represent the longitudinal, transverse and thickness direction of the weld joint. Figure 2 shows the profile of welded joint, which presents a nail shape. The width of the fusion zone is about $5 \mathrm{~mm}$.

\subsection{Contour Residual Stress Measurement}

The contour method, which was developed by Prime [9] and based on the Bueckner's superposition principle, was used to measure the longitudinal residual stress in the middle cross section of the weld joint. It stipulates that when a part containing residual stress is cut into halves along a straight line, the newly created free cut surface will deform owing to the stresses normal to the surface are released. The deformations can be measured and used to calculate the residual stresses prior to the cut. According to Refs. $[9,11,16]$, main procedures of contour method include (1) cutting the part in half to relax the residual stresses inside; (2) measuring the deformations of each of the opposite surface created by the cut; and (3) using the measured deformations to calculate the residual stresses.

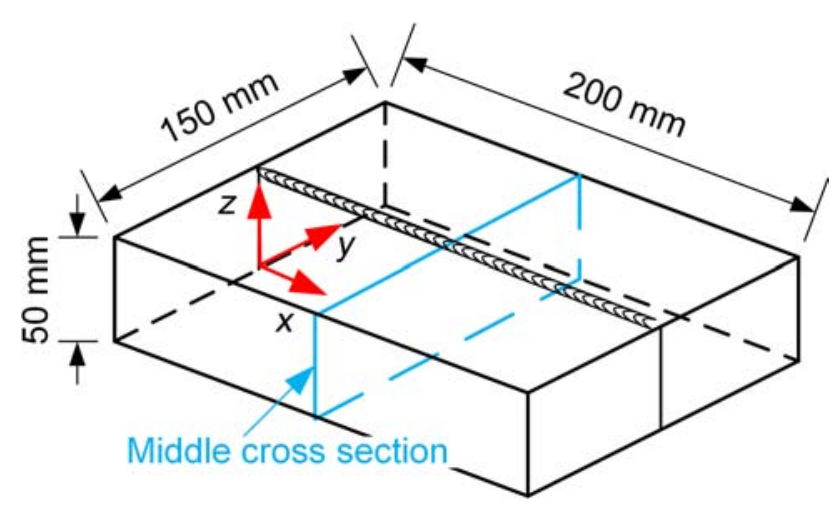

Fig. 1 Schematic diagram of the welded plate 


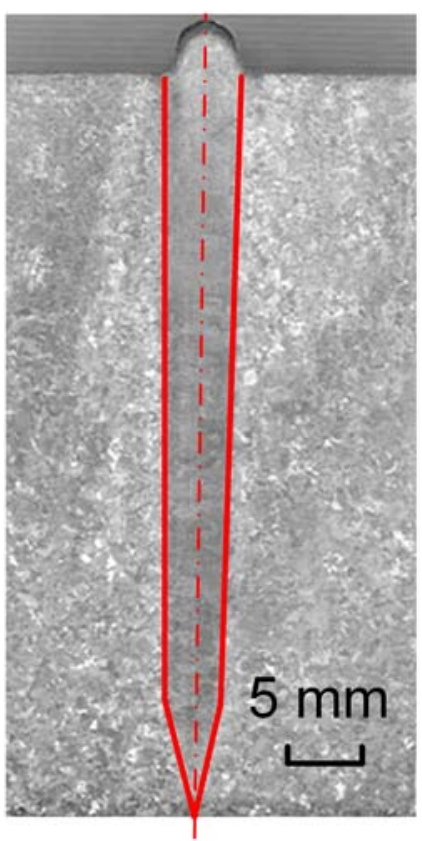

Fig. 2 Optical photograph shows the profile of fusion zone

The specimen was cut in half on the middle cross section (see Fig. 1), by using AgieCharmilles CA20 wired electric discharge machining (EDM) with a $200-\mu \mathrm{m}$-diameter brass wire and $0.2 \mathrm{~mm} / \mathrm{min}$ cutting speed. During the cutting, the clamped specimen was submerged in a temperature-controlled deionized water pool to prevent the additional thermal stress.

The contour of each of the opposing surface created by the cut is then measured by a PRISMO Ultra coordinate measuring machine (CMM) with the nominal accuracy of $\pm 0.6 \mu \mathrm{m}$. Both halves of the specimen were scanned using lattice dimension of $0.5 \mathrm{~mm} \times 0.5 \mathrm{~mm}$. The measured raw data of two opposing surfaces must be averaged and fitted smoothly to remove the influence of shear stress and cutting imperfections [9]. Figure 3 shows the fitted contours of deformation on the cut plane. It can be seen that the contour resemble a ' $\mathrm{V}$ ' shape with a low spot on weld zone, and the peak value is about $180 \mu \mathrm{m}$.

The original stresses normal to the cut plane were calculated by elastically deforming the cut plane into the opposite shape of the deformation measured before. A linear elastic analysis used a 3D finite element model based on ABAQUS software. A single value of the material behaviors was used for all regions in the model. The Young's modulus and the Poisson's ratio are $112 \mathrm{GPa}$ and 0.34 , respectively. The mesh was constructed of one half of the part and used linear hexahedral elements (C3D8R) with $0.5 \mathrm{~mm} \times 0.5 \mathrm{~mm}$ dimensions in the cut plane. The entire model was constrained with two corner nodes to prevent body from moving, as shown in Fig. 4.

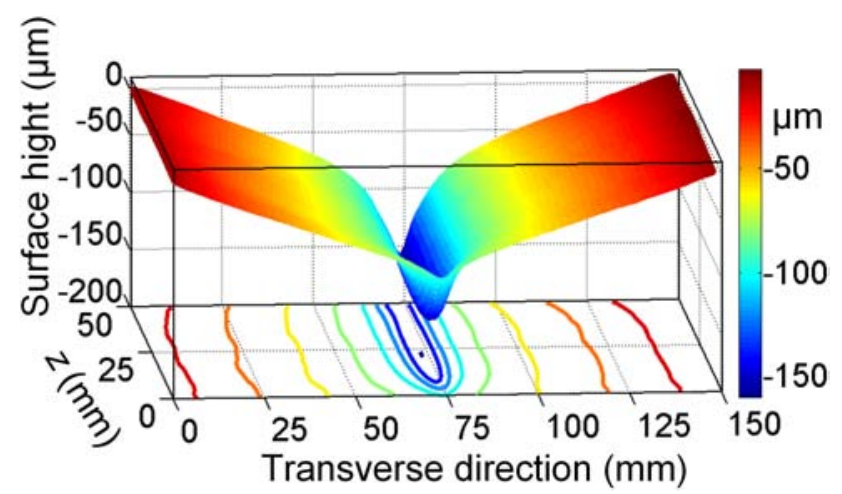

Fig. 3 The fitted deformation of the contour measured

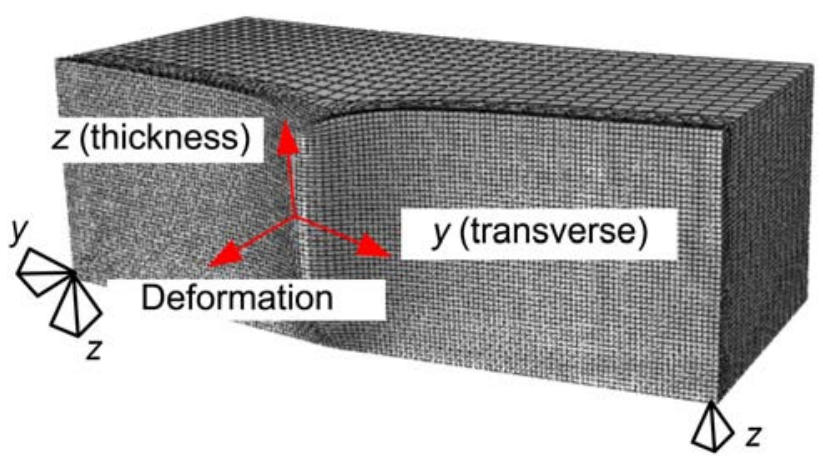

Fig. 4 Finite element model of contour measurement (the deformation is magnified by a factor of 150)

\subsection{Finite Element Modeling}

Only one direction of residual stress normal to the cut surface can be measured in contour method. The residual stresses of all three directions were simulated using FEM. In addition, the simulated results are compared with the previous contour experiment. The 3D finite model in ABAQUS software is shown in Fig. 5. The smallest element is $0.5 \mathrm{~mm} \times 0.25 \mathrm{~mm} \times 0.5 \mathrm{~mm}$, which separately represent the longitudinal, transverse and thickness direction. The mesh has a fine grid in the welding zone with sufficient accuracy for the computed results $[13,14]$. Because of the symmetry of structure, one half of the model is chosen for analysis.

A column-cone combined heat source model was developed to represent the real weld fusion zone, which has the characteristic of large depth-to-width ratio. The heat flux in both column and cone heat source is present as typical Gaussian power distribution in the $x-y$ plane, as shown in Fig. 6.

The heat flux distributions, $q_{\text {cylinder }}(x, y, z)$ and $q_{\mathrm{cone}}(x, y, z)$, are separately represented as follows: 


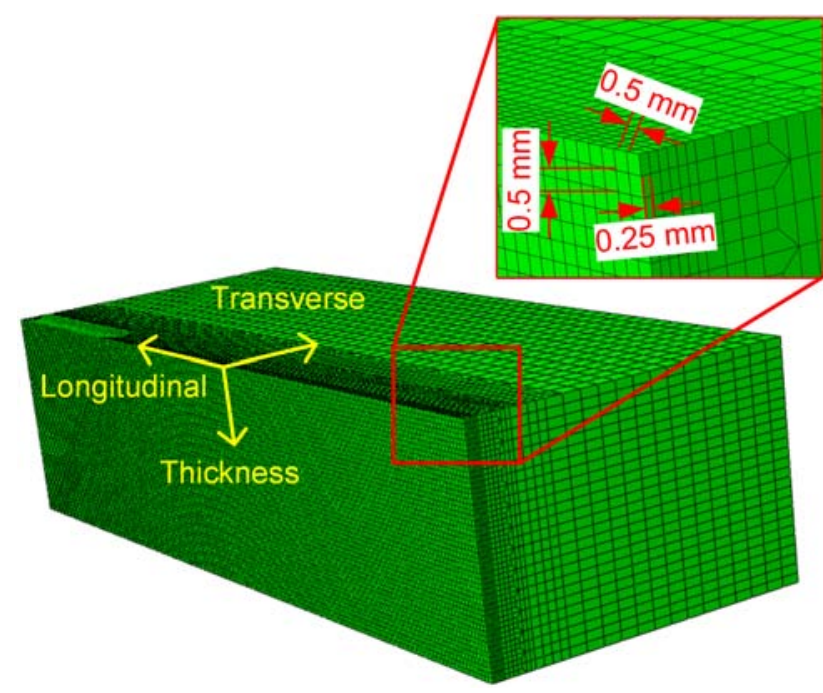

Fig. 5 Finite element model with meshing of the half of the plate

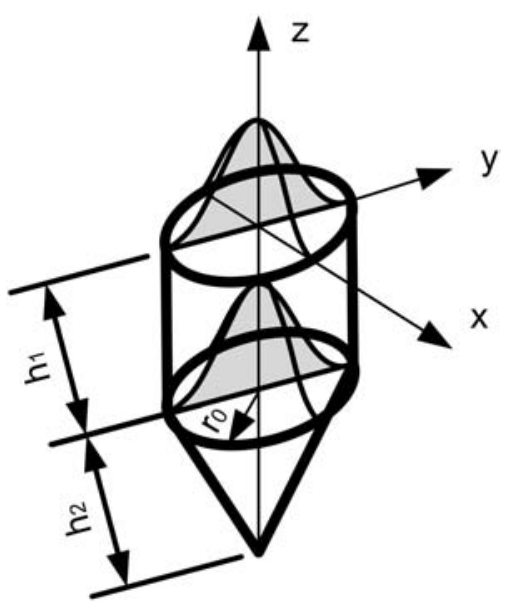

Fig. 6 Schematic illustration of combined heat source model

$$
\begin{aligned}
q_{\text {cylinder }}(x, y, z)= & \frac{3 \alpha Q}{\pi\left(h_{1}+h_{2}\right) r_{0}^{2}} \cdot \exp \left(-k \frac{x^{2}+y^{2}}{r_{0}^{2}}\right), \\
q_{\text {cone }}(x, y, z)= & \frac{3 \beta Q}{\pi\left(h_{1}+h_{2}\right) r_{0}^{2}} \\
& \cdot \exp \left\{\left(-k \frac{x^{2}+y^{2}}{r_{0}^{2}}\right) \cdot\left(1-\varphi \frac{z}{h_{2}}\right)\right\},
\end{aligned}
$$

where $Q$ is the effective heat input power, $r_{0}$ is the equivalent heat source radius at the top surface, $\alpha$ and $\beta$ separately means energy distribution coefficients of cylinder and cone heat source, $h_{1}$ and $h_{2}$ are the depths of cylinder and cone heat source effect, $k$ is concentration coefficient of Gaussian power distribution, $\varphi$ is attenuation coefficient of energy distribution along the depth direction in cone heat source. The unknown parameters are $\alpha, \beta, k$ and $\varphi$. It is necessary to choose these parameters so that the predicted fusion profiles agree with those observed experimentally. Trial simulations based on experience were carried out with the FEM model to determine these unknown parameters. Finally, the optimal parameters were obtained and are given in Table 1.

A similar computational method of temperature and residual stress was reported in Refs. [4, 13-15]. The analysis is divided into two parts to compute the welded residual stress: Firstly, a nonlinear transient thermal analysis was carried out to get the temperature history; secondly, the nonlinear thermal elastic-plastic analysis is performed using the temperature history computed by the first step. In temperature field calculating, only thermal radiation condition and convection heat exchange at the bottom surface are considered because the whole EBW process is completed in vacuum chamber. The part of heat loss which due to radiation obeys the Sten-Boltzman's law, while the part of it that due to the convection obeys the Newton's law. The adiabatic condition is set on the symmetry face. In the stress field calculation, symmetrical boundary condition was applied. In addition, three fixed constraints were added to prevent the body from moving. The thermal conductivity above the melt temperature is given a large value to model the stir effect caused by the fluid flow in the weld pool. The thermal conductivity and the specific heat capacity are used in the thermal transient analysis. The latent heat of fusion was ignored to improve the numerical stability of the thermal analysis, and it is believed that the latent heat has a limited effect on the predicted temperature [17]. The mechanical behavior of material obeys Mises yield criterion, and the bilinear linear kinematic hardening is also included in material model. Above the melt temperature, the mechanical properties are taken into very low values to avoid numerical problems. For example, concerning Young's modulus, for temperatures higher than melt temperature $\left(1660^{\circ} \mathrm{C}\right)$, the considered value must be low enough to ensure that the welding pool cannot transmit significant stresses, but high enough to avoid the ill-conditioning of the stiffness matrix. This small value of Young's modulus is used to avoid convergence issues during the numerical analysis, and it is sufficient to get residual stresses with reasonable accuracy, which is verified in the previous works [18, 19]. The temperature-dependent material properties are shown in Fig. 7.

Table 1 Parameters used in the combined heat source

\begin{tabular}{llllllll}
\hline$Q\left(\mathrm{~kg} \mathrm{~m}^{2} / \mathrm{s}^{3}\right)$ & $r_{0}(\mathrm{~m})$ & $\alpha$ & $\beta$ & $h_{1}(\mathrm{~m})$ & $h_{2}(\mathrm{~m})$ & $k$ & $\varphi$ \\
\hline 17,280 & 0.003 & 0.85 & 0.85 & 0.042 & 0.008 & 3 & 0.18 \\
\hline
\end{tabular}



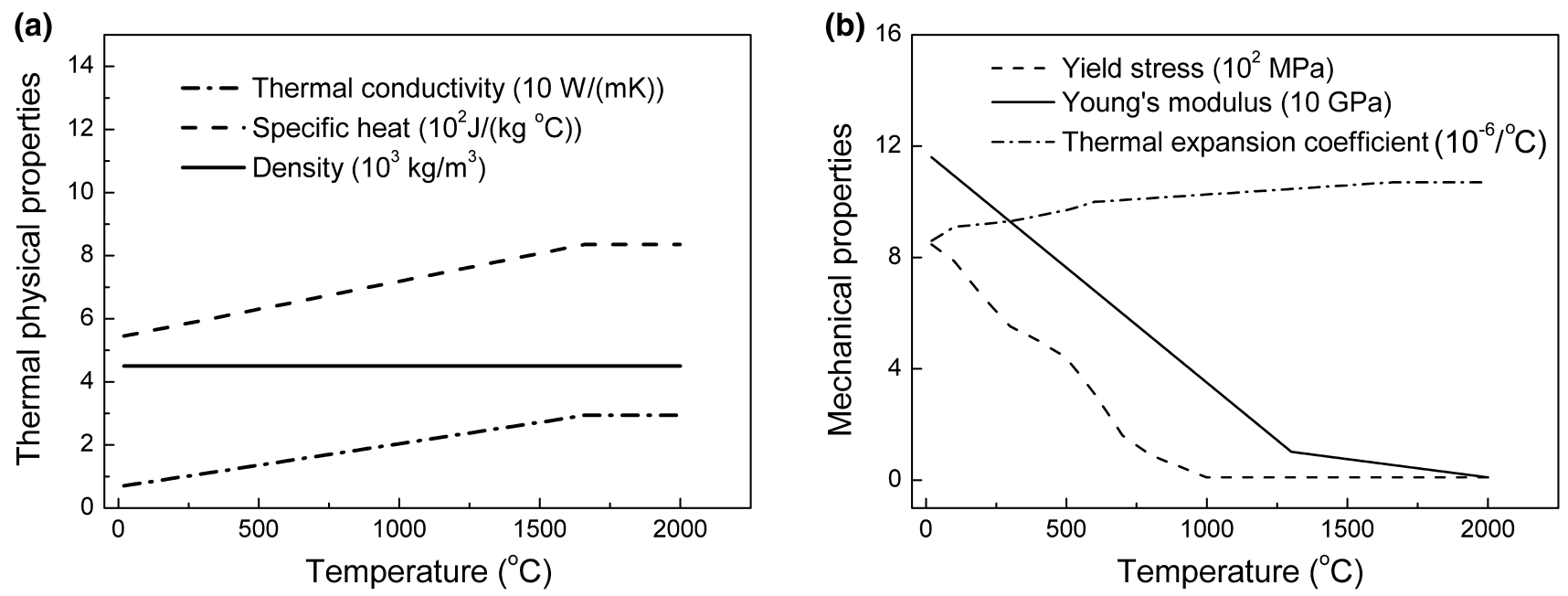

Fig. 7 Temperature-dependent material properties: a thermal physical properties; $\mathbf{b}$ mechanical properties

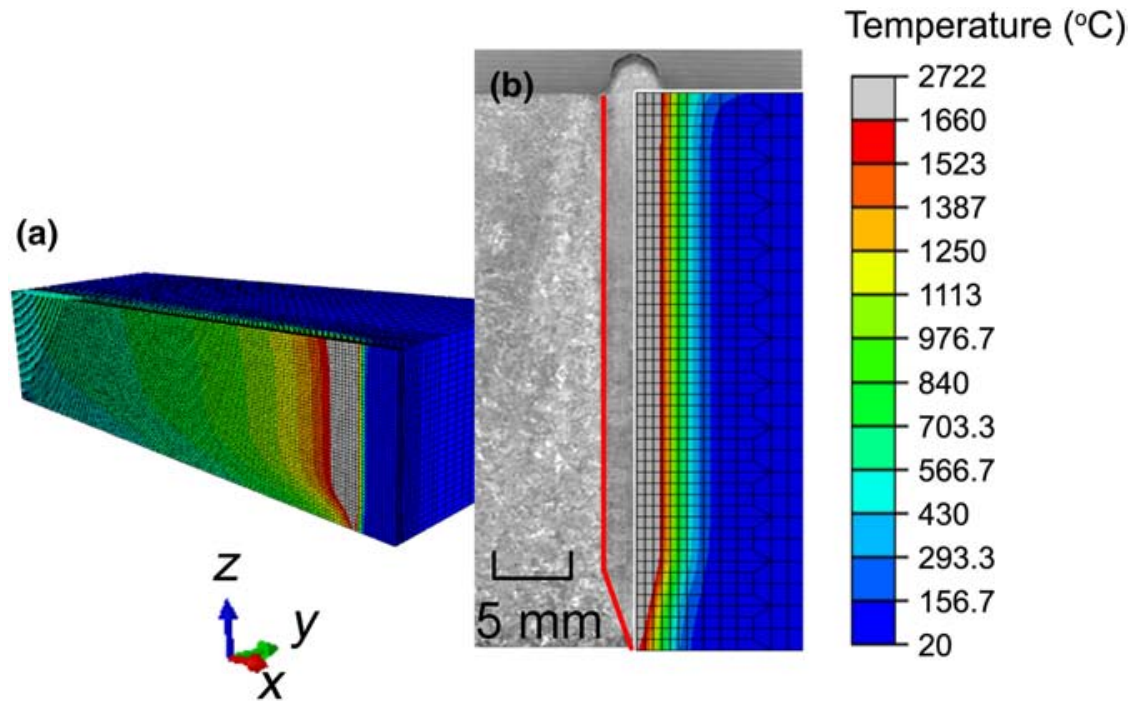

Fig. 8 Simulated temperature field: a 3D temperature field; $\mathbf{b}$ comparison between experimental and predicted fusion profiles

\section{Results and Discussion}

\subsection{Results of Thermal Simulations Using FEM}

Figure 8 shows the temperature field-simulated by the combined cylinder-cone heat source model. It can be seen that the temperature field in the welding process is narrow and most concentrated within $10 \mathrm{~mm}$ of weld zone. The peak value of temperature is about $2700{ }^{\circ} \mathrm{C}$. The fusion zone, for which the temperature is above $1660{ }^{\circ} \mathrm{C}$, resembles a nail shape, as shown in Fig. 8. Compared with the experimental and simulated fusion profiles, they agree well in transverse direction (y-direction), which confirm that the parameters of cylinder and cone heat source in present work could produce an experimentally determined fusion profiles with a reasonable degree of accuracy.

\subsection{Results of Residual Stresses Simulations Using FEM}

Figure 9 shows the longitudinal (welding direction, $x$-direction), transverse (perpendicular to welding direction, $y$-direction) and thickness direction (z-direction) residual stress field-simulated results. Different from thin-wall welded structure, the residual stress along thickness direction cannot be ignored anymore $[6,14]$. The simulated results show that there are large longitudinal tensile residual stresses in the weld zone. The transverse stresses present large compression on top and bottom surfaces of the weld zone but tension inside. The stresses along thickness direction mostly present tensile, and the tensile stresses on both sides are relatively larger than that inside. The final welded residual stress presents triaxial tensile state inside the welded joint. 


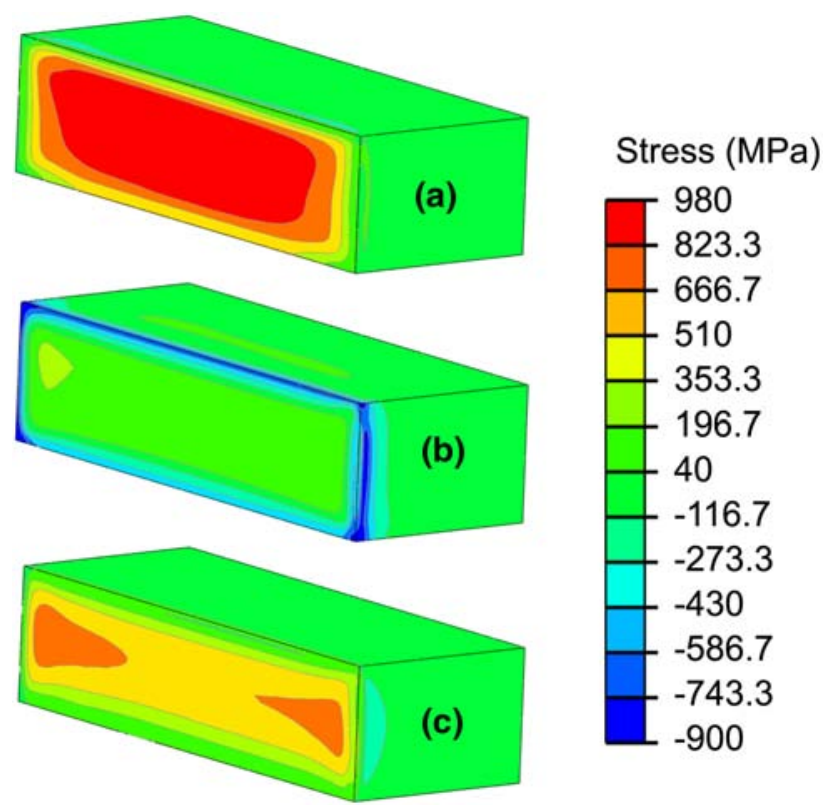

Fig. 9 Welded residual stress simulated by 3D FEM: a longitudinal stress; $\mathbf{b}$ transverse stress; $\mathbf{c}$ thickness stress

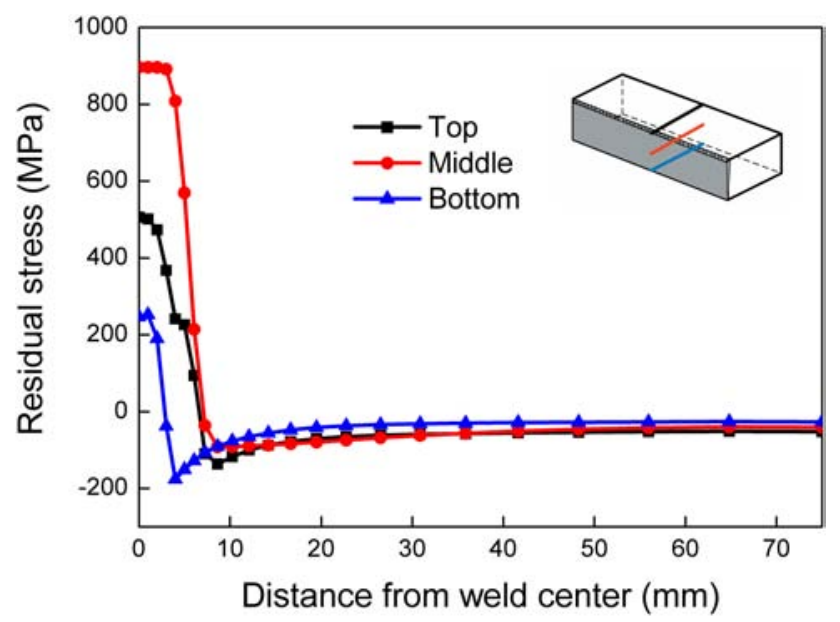

Fig. 10 Longitudinal residual stresses along top, middle and bottom lines

Figure 10 shows the simulated longitudinal residual stresses on the top, middle and bottom lines. It is clear that the highest tensile stress appears on the middle line, and the peak value is about $910 \mathrm{MPa}$ that exceeds the yield strength of material $(890 \mathrm{MPa})$. The tensile stresses on top and bottom surfaces are similar to that on the middle line, but the value is smaller. All tensile longitudinal stresses are concentrated in the weld zone and then transform into compressive stresses around heat affect zone (HAZ) and finally become zero at the region far away from weld. This characteristic indicates that the longitudinal stresses balance each other along transverse direction.

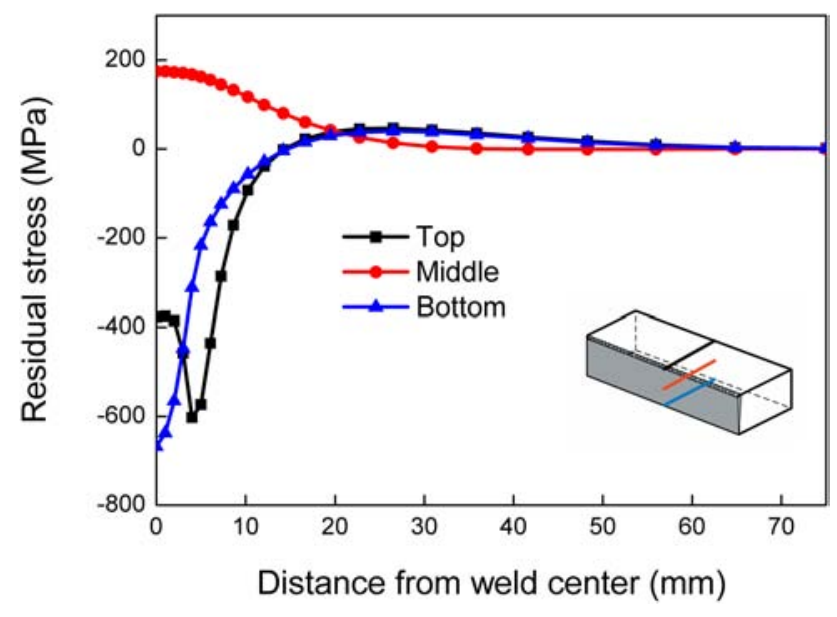

Fig. 11 Transverse residual stresses along top, middle and bottom lines

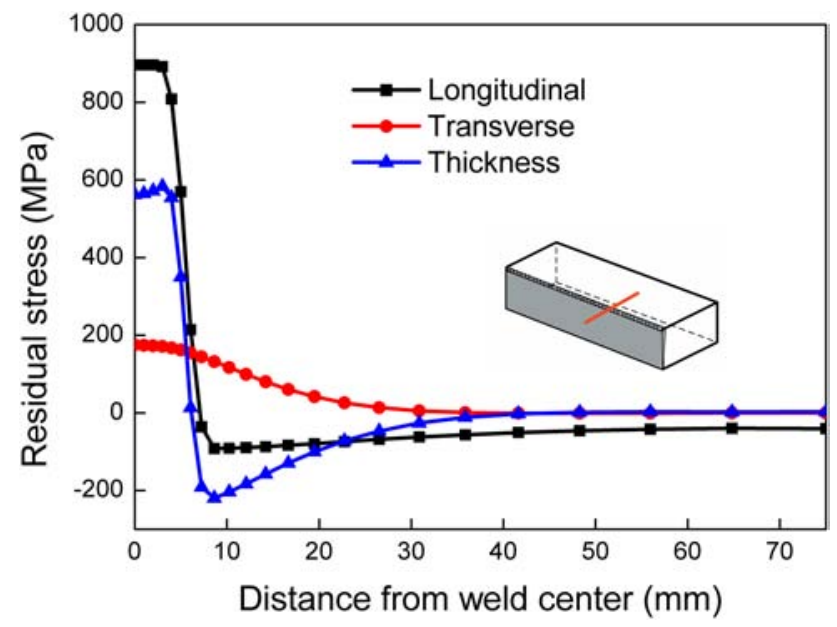

Fig. 12 Comparison of longitudinal, transverse and thickness components of residual stress along middle line

Figure 11 shows the transverse stresses along top, middle and bottom lines. The compressive stress appears on both top and bottom, but the tensile stress is found in the middle. Similar stress distributions were found in the previous work [23]. This can be explained that the interior metal is frozen after the surface metal has been frozen in the cooling stage. The transverse shrinkages of material inside weld joint are restricted to the material on frozen border, which makes the large compressive stress on surface border and the tensile stress inside. The transverse stress change of top line is different from the bottom line in the range near weld center. The transverse stresses on top line shows that the stress falls down first and then rises. It can be contributed to the weld cap on top surface (see Fig. 2). In the FEM simulation, we choose the node results 
(a)

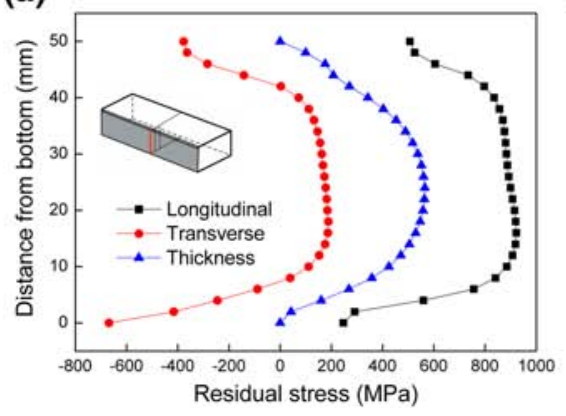

(b)

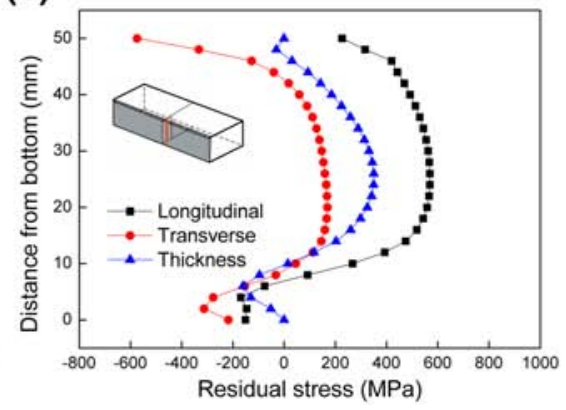

(c)

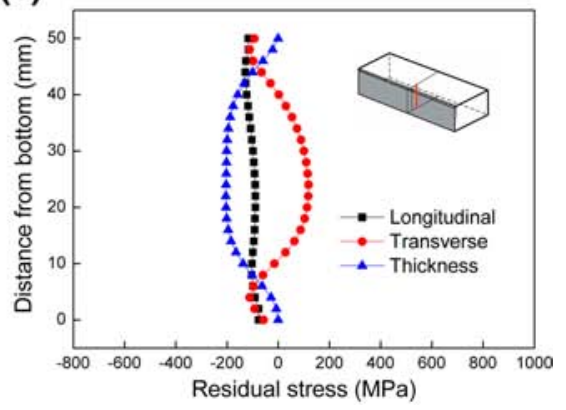

Fig. 13 Comparison of residual stresses in different directions: a along weld center line; $\mathbf{b}$ along the line with 5-mm distances from weld center; c along the line with $10-\mathrm{mm}$ distances from weld center

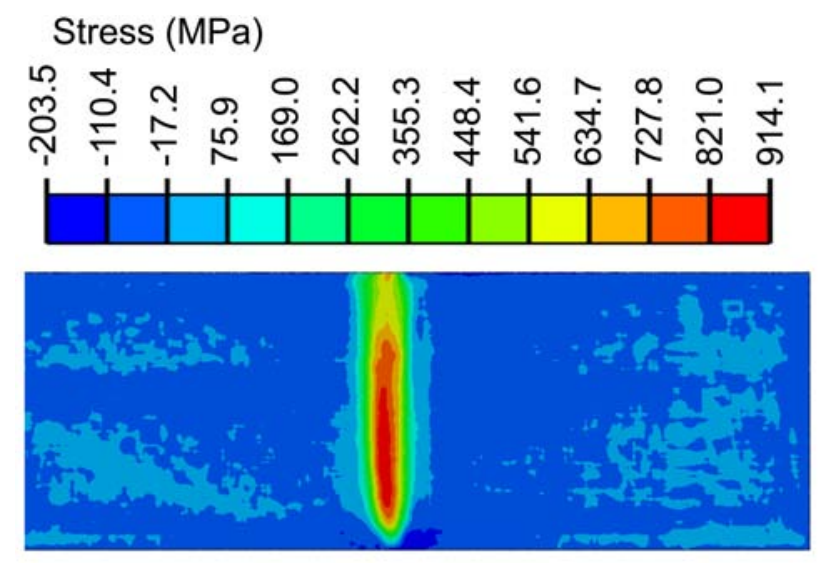

Fig. 14 Mapping longitudinal stresses on the cut plane of the contour method
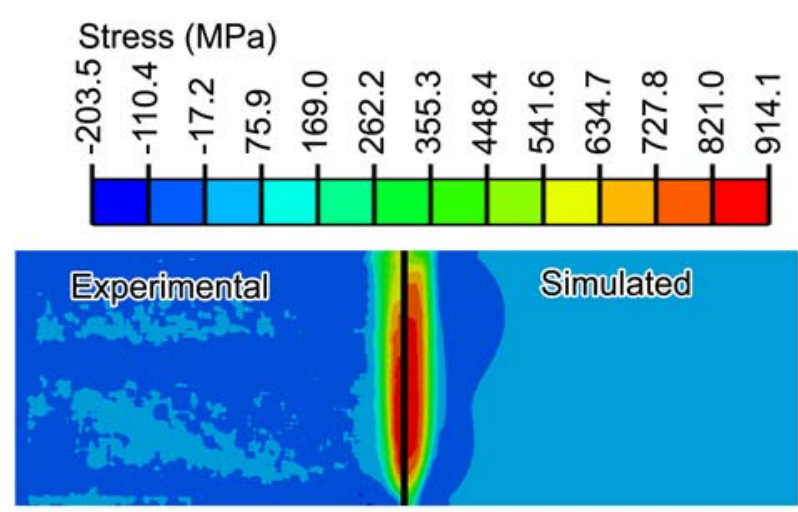

Fig. 15 Comparison of the simulated and experimental 2D maps of longitudinal residual stress

on top line, which is located on weld cap borders. The weld cap reduces the constraint on top surface of weld, which makes the compression near weld center relax.

Figure 12 shows the longitudinal, transverse and thickness components of residual stress along middle line. It can be seen that in the middle of the weld joint, the residual stress presents a tri-tensile state, and the thickness component of residual stress is the smallest one compared with

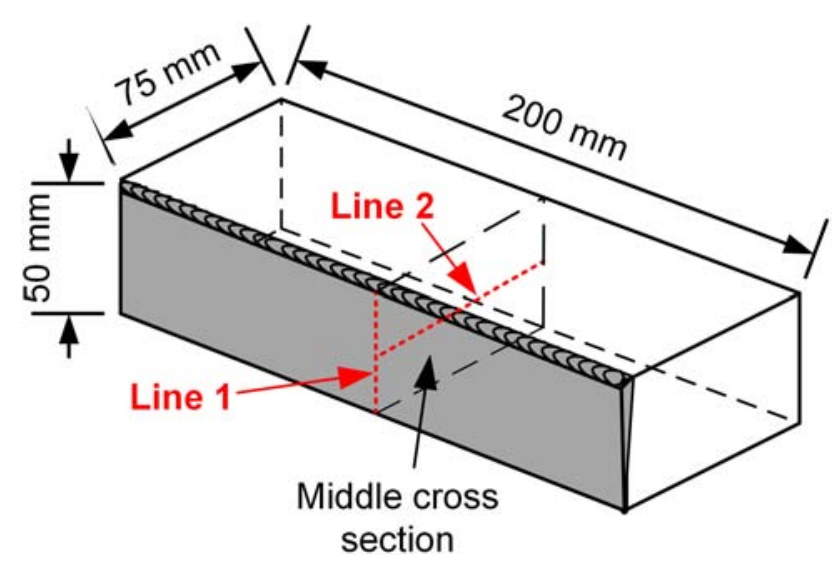

Fig. 16 Schematic diagram of the two lines for residual stress analysis

the other two components. This can be explained that the magnitude of residual stress depends on the state of constraints. The longitudinal constraint is the largest in welding, and the constraint in thickness component is the smallest in the same welding condition.

Unlike thin-wall weld, the change of residual stress from top to bottom cannot be ignored in the present studies. The simulated residual stresses along the weld center, 5-mm distance from the weld center (around HAZ), and 10-mm distance from the weld center (far away from weld) are separately shown in Fig. 13. It can be seen from Fig. 13a that all residual stresses are larger in middle and lower on either side along the weld center. This characteristic has been maintained to HAZ (5-mm distances from weld center), as shown in Fig. 13b. At the region that is far away from weld (10-mm distances from weld center), all residual stress reduce to about zero, which are shown in Fig. 13c.

\subsection{Comparison of Simulated and Experimental Results}

In contour method, all the measured stresses are normal to the cut plane. In other words, the longitudinal stresses $(x-$ 
(a)

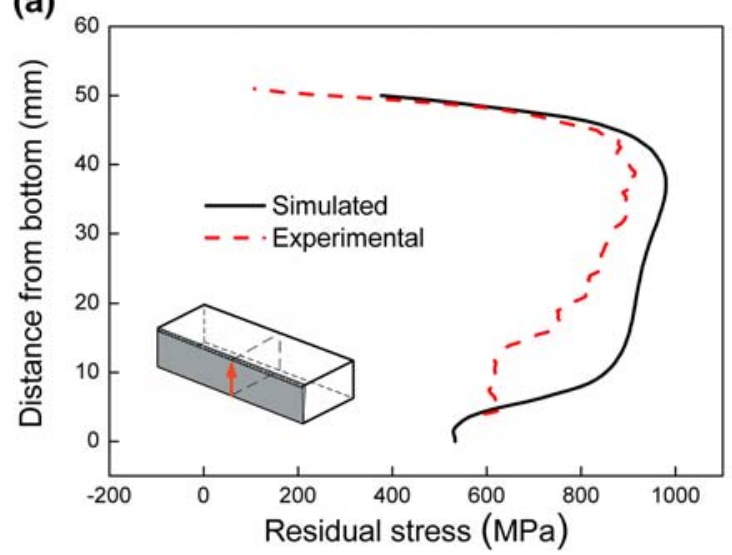

(b)

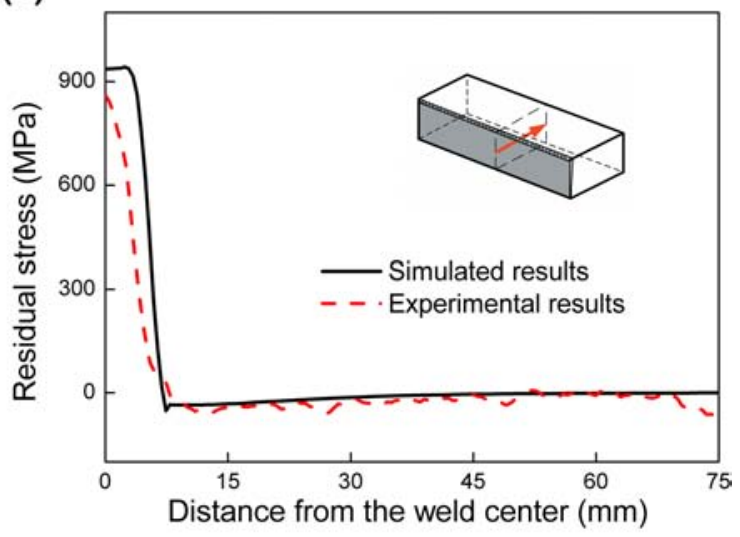

Fig. 17 Longitudinal residual stress along the two lines in Fig. 16: a line 1; b line 2

direction) are measured in the present studies. Figure 14 shows the longitudinal residual stress in middle cross section measured by contour method. It can be seen that the longitudinal stress is entirely tensile stress inside the weld joint except that a small compressive stress zone is presented on the bottom side. The longitudinal stresses are obviously different at different depths of weld zone. The longitudinal residual stress reaches a maximum tensile stress about $914 \mathrm{MPa}$ in the middle of weld zone, which exceeds the yield strength of $890 \mathrm{MPa}$.

Considering the uncertainty of the welding simulation [7], the simulated results were confirmed by experimental data from contour method. Figure 15 shows the comparison of simulated and experimental results, both of them agree well with each other.

Two lines in the welded component are chosen to compare the simulated results and experiment better, as seen in Fig. 16. The line 1 is weld centerline, and the line 2 is the middle of thickness. The simulated and experimental residual stresses are separately compared along these two lines, as shown in Fig. 17.

From Fig. 17, it can be seen that the simulated tensile stresses are slightly larger than the experimental results. One reason for over simulated stresses could be that the experimental results may be the mean stress at a certain area rather than at the node of FEM. Another reason for the differences could be that there are some random errors such as over cutting during the EDM. As shown in Fig. 17a, the measured longitudinal stress near top surface (line one) is near zero, which is different from FEA. It can be explained that there may be some cutting-related issues associated with EDM near the border surfaces in present work. According to Refs. [20, 21], the stresses at the cut tip during the cutting procedure cause the material to deform, and some bulge errors tend to cause stress profiles to show a reduced peak stress magnitude. In general, contour results are uncertain near the edges of the cut and should not be reported in that region unless special care is taken to get better results there [22].

\section{Conclusions}

(1) The combined cylinder-cone heat source model is an effective model for simulating the temperature field of the Ti-6Al-4V alloy plates (50 mm thick) of EBW. The simulated results show that the weld fusion zone appears nail-shaped, and the width of it is $5 \mathrm{~mm}$.

(2) The welded residual stress in thick titanium plate is calculated using sequenced coupled thermal elasticplastic analysis method based on ABAQUS software. The longitudinal stress, transverse stress and thickness components of stress are present, respectively.

(3) The simulated results of FEM are calibrated by contour experiment. Both simulated and experimental results show that there is large longitudinal tensile stress inside the weld joint, and its value is $910 \mathrm{MPa}$, which exceed yield strength of the material.

(4) The transverse residual stress is compression near the top and the bottom surfaces, rather than tension inside. The residual stresses are in tri-tension state in the middle of the weld joint.

Acknowledgments This work was financially supported by the National Natural Science Foundation of China (No. 50935008).

\section{References}

[1] R.R. Boyer, Mater. Sci. Eng. A 213, 103 (1996)

[2] A. De, T. DebRoy, Sci. Technol. Weld. Join. 16, 204 (2011)

[3] J. Goldak, M. Bibby, J. Moore, R. House, B. Patel, Metall. Trans. B 17, 587 (1985) 
[4] D. Deng, Y. Tong, N. Ma, H. Murakawa, Acta Metall. Sin. (Engl. Lett.) 26, 333 (2013)

[5] P. Lacki, K. Adamus, P. Wieczorek, Comput. Mater. Sci. 94, 17 (2014)

[6] C. Liu, J. Zhang, J. Niu, Rare. Met. Mater. Eng. 38, 1317 (2009). (in Chinese)

[7] P. Michaleris, Sci. Technol. Weld. Join. 16, 722 (2011)

[8] N.S. Rossini, M. Dassisti, K.Y. Benyounis, A.G. Olabi, Mater. Des. 35, 572 (2012)

[9] M.B. Prime, J. Eng. Mater. Technol. 123, 162 (2001)

[10] A. Kundu, P.J. Bouchard, S. Kumar, K.A. Venkata, J.A. Francis, A. Paradowska, G.K. Dey, C.E. Truman, Sci. Technol. Weld. Join. 18, 70 (2013)

[11] C. Liu, X. Yi, Mater. Des. 46, 366 (2013)

[12] W.X. Yu, M.Q. Li, J. Luo, Rare Met. Mater. Eng. 38, 19 (2009). (in Chinese)

[13] A. Lundback, H. Runnemalm, Sci. Technol. Weld. Join. 10, 717 (2005)

[14] C. Liu, B. WU, J.X. Zhang. Metall. Mater. Trans. B 41, 1129 (2010)
[15] G. Xu, C.S. Wu, X.Z. Ma, X.Y. Wang, Acta Metall. Sin. (Engl. Lett.) 26, 352 (2013)

[16] M.B. Prime, T. Gnaupel-Herold, J.A. Baumann, R.J. Lederich, D.M. Bowden, R.J. Sebring, Acta Mater. 54, 4013 (2006)

[17] D.J. Smith, P.J. Bouchard, D. George, J. Strain Anal. 35, 287 (2000)

[18] O. Asserin, A. Loredo, M. Petelet, B. looss, Finite Elem. Anal. Des. 47, 1004 (2011)

[19] A.A. Bhatti, Z. Barsoum, H. Murakawa, I. Barsoum, Mater. Des. 65, 878 (2015)

[20] M.B. Prime, A.L. Kastengren, Exp. Appl. Mech. 6, 233 (2011)

[21] F. Hosseinzadeh, P. Ledgard, P.J. Bouchard, Exp. Mech. 53, 829 (2013)

[22] M.B. Toparli, M.E. Fitzpatrick, S. Gungor, Exp. Mech. 53, 1705 (2013)

[23] C. Liu, J.X. Zhang, B. Wu, S.L. Gong, Mater. Des. 34, 609 (2012) 